\title{
Le lexique en français et en yoruba : Modes d'enrichissement
}

\author{
Tajudeen Abodunrin OSUNNIRAN (Ph.D.) \\ Department of Languages and Literary Studies, Kwara State University, Malete, Kwara State.
}

\begin{abstract}
Résumé : La langue, face à la nature évolutive de la société au sein de laquelle elle trouve sa raison d'être, se doit aussi d'évoluer et de changer au cours du temps afin d'être en mesure de jouer pleinement son rôle d'outil de communication. Parmi la gamme de changements que peut subir une langue, nous sommes concerné dans cette communication par les changements qui affectent le lexique, c'est-à-dire les mots de la langue. Nous nous proposons d'exposer les mécanismes linguistiques à la disposition du français et du yoruba dans l'enrichissement de leur lexique afin de mieux s'adapter aux besoins évolutifs de leurs usagers. C'est une étude purement linguistique. La démarche est celle de l'analyse contrastive. L'objectif est de s'intéresser aux similarités et aux différences entre deux systèmes linguistiques dans le but de contribuer à la connaissance des structures des langues et à l'appréciation de la diversité qui peuvent exister entre elles.

Mots-clés : lexique, vocabulaire, enrichissement, procédés morphologiques
\end{abstract}

\section{Introduction}

Lexique et vocabulaire sont deux termes employés en référence à l'ensemble de l'opération de la mise en mots des unités de première articulation dans toute langue. Les deux termes prêtent souvent à confusion. Avant toute chose, il nous est donc nécessaire de faire la lumière sur ces termes, d'ailleurs indispensables à toute discussion en lexicologie.

Pour Phelizon (1976 : 130) citant Wagner, le lexique est l'ensemble de mots au moyen desquels les membres d'une communauté linguistique communiquent. Mounin (1974 : 203), pour sa part, présente le lexique comme l'ensemble des unités significatives d'une langue donnée, à un moment donné de son histoire. De ces deux définitions, on peut présenter le lexique comme étant l'ensemble des mots à la disposition d'une langue donnée et qui sert de moyen de communication à ses membres. Personne ne peut quantifier les mots d'une langue. C'est un ensemble ouvert. Chacun ne vient qu'y puiser ce qui lui sera nécessaire pour sa communication et cela constitue son lexique individuel (ensemble des mots qu'il peut employer et comprendre) puisé dans un lexique général ou global.

Le vocabulaire, pour sa part, se présente comme l'ensemble des mots connus et effectivement employés par un locuteur ou un groupe social. Dans ce sens, le lexique appartient au domaine du virtuel (ensemble des mots qui existent ou qui peuvent exister) et le vocabulaire au domaine du concret (ensemble de mots effectivement utilisé dans tel ou tel acte de parole). Ainsi, le vocabulaire est un sous-ensemble du lexique individuel, lui-même un sous-ensemble d'un lexique général. Genouvrier et Peytard (1970: 181) vont dans le même sens quand ils affirment que : "Vocabulaire et lexique sont en rapport d'inclusion : le vocabulaire est toujours une partie, de dimensions variables, selon le moment et les sollicitations, du lexique individuel, luimême partie du lexique global'". Tous les locuteurs d'une langue partagent une masse d'unités lexicales. Aucun locuteur n'en possède toutes, mais ensemble, les vocabulaires combinés définissent une unité supérieure qui existe au niveau de la communauté ; c'est cette unité supérieure que nous appelons le lexique.

Le vocabulaire, et par extension le lexique, n'est cependant pas statique; il se renouvelle à chaque instant. Les mots naissent, vivent et disparaissent de l'usage selon les réalités sociales en vogue. Quand de nouveaux mots apparaissent ou quand d'anciens mots reviennent pour de nouveaux emplois, on dit que la langue s'enrichit. C'est de cet enrichissement lexical qu'il sera question dans cette communication et notre préoccupation est de savoir comment il a lieu en français et en yoruba. Cette étude s'inscrit dans le cadre de la linguistique théorique et adoptant l'esprit de l'analyse contrastive, elle se veut une contribution à la connaissance des structures des langues et à l'appréciation de la diversité qui peut exister entre elles.

\section{Le lexique du français : Modes d'enrichissement}

Le lexique du français a une constitution hétéroclite ; il s'est constitué à travers des apports d'origines diverses. Pour Picoche et Marchello-Nizia (1991 : 323), "Selon les calculs de D. Messner, les mots français seraient d'origine latine à $86,53 \%$, germanique à $1,35 \%$, scandinave à $0,12 \%$, celtique à $0,08 \%$, le reste consistant en emprunts". En poussant le calcul plus loin, on pourrait estimer à 13,27\% le nombre d'emprunts qui ont contribué à la constitution du lexique français. Cette assertion trouve aussi confirmation chez Huot (2001 : 5) qui atteste que “... pour plus de $80 \%$, le stock de mots constituant le lexique du français moderne provient du latin” et plus loin, “... on estime néanmoins à environ $13 \%$ du vocabulaire total les mots empruntés à des 
langues étrangères" (2001: 14). Mais ce lexique ainsi constitué n'est pas resté figé, au cours du temps, il s'élargit pour inclure de nouveaux mots pour de nouveaux concepts afin de s'adapter aux évolutions du monde de l'heure. Dans cette sous-section, nous ferons un parcours des outils à la disposition du français pour enrichir son lexique en réponse à cette exigence.

\section{A. L'enrichissement lexical par des procédés morphologiques}

De nouveaux mots peuvent être creés en employant des procédés morphologiques. Ici, il s'agit de créer de nouveaux mots à partir de ceux existants en employant les méthodes comme la dérivation, la composition, l'abrègement, la siglaison, etc.

\section{(a) La dérivation}

La dérivation est un procédé qui consiste à obtenir un nouveau mot avec un nouveau sens par l'ajout d'un affixe à la base d'un mot déjà existant. La base lexicale à laquelle s'ajoute un affixe peut être un verbe, un adjectif ou un nom et peut exister de façon autonome (base libre) ou non (base liée). L'affixe n'a pas une existence indépendante c'est-à-dire qu'il ne peut à lui seul constituer un mot (en exemple, les affixes 'pré' et 'ment' dans 'prédire' et 'poliment'). Selon qu'il se place devant ou derrière une base lexicale, il prendra le nom de préfixe et de suffixe respectivement. On peut alors parler de dérivation préfixale ou préfixation (ajout de préfixe à une base) ou de dérivation suffixale ou suffixation (ajout de suffixe à une base).

Préfixation $=$ préfixe + base $=$ mot dérivé

$($ Ex : dé- + charge $=$ décharge $($ charge, base libre $))$

Suffixation $=$ base + suffixe $=$ mot dérivé

$(E x:$ ferme + -ture $=$ fermeture (ferme, base liée, vient de fermer)

Les affixes ne s'emploient pas au hasard. Il y a des règles à suivre, des contraintes linguistiques à respecter : les affixes de la langue sélectionnent leurs bases (nom, adjectif, verbe) et ils sont utilisés selon la contribution sémantique désirée. Le préfixe a une valeur surtout sémantique, il n'entraîne pas de changement de classe grammaticale alors que le suffixe peut entraîner un changement de classe grammaticale. Ce tableau nous fournit quelques exemples :

\begin{tabular}{|c|c|c|c|}
\hline Base & Suffixe & Nouveau mot obtenu & Contribution sémantique \\
\hline $\begin{array}{l}\text { Verbale (V) } \\
\text { chant(er) } \\
\text { Nominale (N) } \\
\text { courag(e) } \\
\text { Adjectivale (Adj) } \\
\text { lent }\end{array}$ & $\begin{array}{l}+ \text { eur } \\
\text { chant+eur } \\
+ \text { eux } \\
\text { courag+eux } \\
+ \text { ment } \\
\text { lente+ment }\end{array}$ & $\begin{array}{l}\text { Nom } \\
\text { chanteur } \\
\text { Adjectif } \\
\text { courageux } \\
\text { Adverbe } \\
\text { lentement }\end{array}$ & $\begin{array}{l}\text { celui qui } \mathbf{V} \\
\text { celui qui chante } \\
\text { qui a le caractère de } \mathbf{N} \\
\text { qui a le courage } \\
\text { de manière Adj } \\
\text { de manière lente }\end{array}$ \\
\hline Préfixe & Base & Nouveau mot obtenu & Contribution sémantique \\
\hline $\begin{array}{l}\text { ré }+ \\
\text { ré }+ \\
\text { im/in }+ \\
\text { im+ } \\
\text { pré }+ \\
\text { pré+ }\end{array}$ & $\begin{array}{l}\text { Verbale } \\
\text { faire } \\
\text { Adjectivale } \\
\text { possible } \\
\text { Nominale } \\
\text { histoire }\end{array}$ & $\begin{array}{l}\text { Verbe } \\
\text { refaire } \\
\text { Adjectif } \\
\text { impossible } \\
\text { Nom } \\
\text { préhistoire }\end{array}$ & $\begin{array}{l}\text { la répétition } \\
\text { faire de nouveau } \\
\text { la négation } \\
\text { ne pas être possible } \\
\text { avant } \mathbf{N} \\
\text { avant l'histoire }\end{array}$ \\
\hline
\end{tabular}

Quand il y a besoin d'obtenir une unité complexe par usage de préfixe et/ou de suffixe, il faut faire attention à l'ordre (quel affixe pour quelle base ?) tout au long du processus. Voyons cet exemple :

Le mot réévaluation : $\quad *$ évaluer - évaluation - réévaluation évaluer - réévaluer - réévaluation

Le deuxième ordre de dérivation est possible car 're' ne s'ajoute qu'à un verbe.

Il existe aussi la dérivation parasynthétique qui consiste à obtenir un nouveau mot par l'ajout simultané d'un préfixe et d'un suffixe à une base donnée. Un trait à retenir avec cette forme de construction est que le mot construit ne peut exister sans le préfixe et le suffixe à la fois.

Dérivation parasynthétique : $\quad$ préfixe + base + suffixe $*($ préfixe + base $) *($ base + suffixe $)$

Exemples : intonation $=$ in + ton + ation $\quad(*$ inton et *tonation n'existent pas $)$ emprisonner $=\mathrm{em}+\operatorname{prison}(\mathrm{n})+\mathrm{er} \quad(*$ emprison et $*$ prisonner n'existent pas $)$

Il faut aussi faire mention de la dérivation régressive. Cette fois-ci, on forme un nouveau mot en supprimant un suffixe. Dérivation régressive $=$ mot - suffixe. Ex : voler $/ \mathrm{vol}$, offrir/offre, marcher $/ \mathrm{marche}$, galoper/galop, nager/nage, dormir/dorme, accorder/accord, plier/pli, refuser/refus, visiter/visite, etc. Ces exemples-ci sont des cas de création lexicale par déverbalisation (la chute du morphème de l'infinitif) grâce à la 
dérivation régressive.

Nous avons aussi la dérivation impropre aussi connue sous le nom de conversion. Elle est impropre en ce sens qu'elle n'implique pas l'adjonction d'affixe à une base mais plutôt un changement de classe grammaticale. On la présente de ce fait comme une substitution de classe grammaticale (surtout entre le nom et l'adjectif). Ex : sourire (verbe) / le sourire (nom), vrai (adjectif) / le vrai (nom), moi (pronom) / le moi (nom), etc.

Pour clore cette section, il convient de remarquer que le français se sert de ce procédé de dérivation pour avoir une importante quantité de néologismes. Pour mesurer l'importance de ce procédé, écoutons cette remarque de Fagyal et al. (2006: 106) :

A study of the words listed under the letter A of the Petit Larousse
dictionary found that sixty-eight percent of those lexical items were formed
by the addition of derivational suffixes (...). This gives some indications of
the importance of derivation as a process of lexical creation.

Il existe des mots créés par ce processus qui sont déjà attestés dans l'usage mais il existe également de nombreux mots possibles qui pourraient un jour se tailler une place parmi les mots existants.

\section{(b) La composition}

La composition, en tant que procédé morphologique de création lexicale, consiste en la juxtaposition d'au moins deux bases lexicales pour former un nouveau mot. Martinet $(1970: 134)$ explique la différence entre la composition et la dérivation en ces termes :

La différence entre composition et dérivation se résume assez bien en disant que les monèmes qui forment un composé existent ailleurs que dans des composés, tandis que, de ceux qui entrent dans un dérivé, il y en a un qui n'existe que dans les dérivés et qu'on appelle traditionnellement un affixe.

La différence entre 'dérivation' et 'composition' réside dans le fait que pour la dérivation, on a adjonction des affixes qui n'ont pas d'autonomie syntaxique à une base lexicale alors que les éléments qui se combinent dans la composition ont chacun leur autonomie syntaxique. On a donc dans ce cas la juxtaposition de deux bases lexicales $\left(M_{1}\right.$ et $\left.M_{2}\right)$ pour former un nouveau mot $\left(M_{3}\right)$. Composition $=\left(M_{1}\right)+\left(M_{2}\right)=\left(M_{3}\right)$, ex : porte $\left(\mathrm{M}_{1}\right)+$ feuille $\left(\mathrm{M}_{2}\right)=$ portefeuille $\left(\mathrm{M}_{3}\right)$.

Selon la nature des bases lexicales qui se combinent pour donner le mot composé, on distingue en français la composition savante et la composition populaire. On parle de composition savante quand les bases lexicales proviennent du latin ou du grec. Cette forme de création lexicale pourrait être qualifiée d'“emprunts par composition lexicale". Ex $:$ carni + vore $=$ carnivore $;$ biblio + thèque $=$ bibliothèque $;$ bio + logie $=$ biologie . Mais, souvent, on a une composition à caractère hybride c'est-à-dire fait d'une base latine ou grecque et d'une base française : auto $($ grecque $)+$ punition $($ française $)=$ autopunition $;$ auto $($ grecque $)+$ route $($ française $)=$ autoroute ; néo (grecque) + capitalisme (française) = néocapitalisme.

On parle de composition populaire quand la composition se fait par juxtaposition de mots français : bon + homme $=$ bonhomme $;$ chou + fleur $=$ chou-fleur $;$ coupe + ongles $=$ coupe-ongles. Presque tous les mots obtenus par composition en français sont des noms. Ces noms sont cependant obtenus à travers divers types de combinaisons dont voici quelques exemples :

- Nom + Nom $=$ chirurgien-dentiste, bateau-mouche, roman-photo, autobus, etc.

- $\quad \mathrm{Nom}+$ Adjectif $=$ feu-rouge, libre-échange, sang-froid, etc.

- $\quad$ Adjectif + Nom $=$ rouge-gorge, pur-sang, gentilhomme, etc.

- Verbe + Nom $=$ gratte-ciel, porte-bagage, porte-manteau, chauffe-eau, lave-vaisselle, etc.

- Verbe + Verbe $=$ savoir-faire, pousse-pousse, laisser-faire, etc.

- Préposition + Nom = à-côté, sans-cœur, en-tête, etc.

- Adverbe + Nom $=$ arrière-pensée, arrière-boutique, etc.

- Nom + Préposition + Nom $=$ arme à feu, machine à coudre, pomme de terre, etc.

A part la dérivation et la composition, il existe d'autres sources internes d'enrichissement du lexique en français dont les suivantes :

\section{(c) La troncation}

C'est un procédé qu'on rencontre surtout dans la langue parlée. Il consiste à créer un nouveau mot en abrégeant un mot par suppression d'une ou de plusieurs de ses syllabes. On appelle aussi ce procédé la réduction ou l'abrègement. En français, c'est généralement la fin du mot qui tombe. Exemples : une automobile - une auto ; un dictionnaire - un dico ; un laboratoire - un labo ; un professeur - un prof. Quand c'est la finale du mot 
qui est tronquée comme dans les exemples ci-dessus, on a une troncation par apocope. Mais quand c'est l'initiale du mot qui est tronquée, on parle plutôt de troncation par aphérèse : capitaine - 'pitaine, monsieur 'sieur.

Ce procédé est source de création de mots valises - mots obtenus par juxtaposition de deux mots tronqués : produit $($ pro $)+$ logiciel $($ giciel $)=$ progiciel ; français $($ fran $)+$ anglais $($ glais $)=$ franglais ; hélicoptère (héli) + aéroport (port) $=$ héliport.

\section{(d) La siglaison et l'acronymisation}

La siglaison et l'acronymisation sont des procédés qui consistent à prendre une ou plusieurs lettres du début de chaque mot d'une locution pour créer un nouveau mot. Picoche et Marchello-Nizia (1991: 362) remarquent qu'“après la Deuxième Guerre Mondiale, le sigle devient un phénomène de créativité lexicale d'une ampleur sans précédent". Exemples :

$$
\begin{aligned}
& \text { Organisation des Nations Unies }=\text { ONU } \\
& \text { Organisation du Traité Atlantique Nord }=\text { OTAN } \\
& \text { Syndrome Immuno-Deficience Acquise }=\text { SIDA }
\end{aligned}
$$

Selon la manière dont ces mots sont prononcés, on parlera de sigles ou d'acronymes. Les sigles sont des mots prononcés avec les noms des lettres qui composent le mot (VTT [vetete] = Vélo Tout Terrain ; SDF

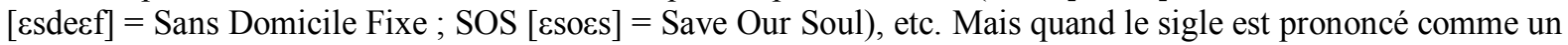
mot ordinaire, il prend le nom d'acronyme : ONU [ony], SIDA [sida], OTAN [otã], RADAR [RadaR] (Radio Detection And Ranging), etc. Un acronyme peut être lexicalisé et dans ce cas, il peut être accentué ou varier en nombre : des radars, des lasers, les cedex, etc. Les acronymes peuvent servir de racines dans la création de nouveaux mots à travers le processus de dérivation. Exemples : L'ONU - Un onusien, une onusienne ; SIDA Un sidéen, une sidéenne ; RADAR - Radaniser

\section{(e) La réduplication}

Ce procédé de création lexicale en français se retrouve dans le langage des enfants. Il consiste en la réduplication de la première syllabe d'un mot : jouet - joujou, chou - chouchou, dormir - dodo, folle - fofolle, père - pépère, mère - mémère, etc.

\section{B. L'enrichissement lexical par le sens}

La langue peut enrichir son lexique en attribuant à des mots anciens de nouveaux sens. Ainsi, un mot ancien renaît avec un nouveau sens pour un nouvel emploi. Des mots anciens qui étaient supposés disparaître avec la disparition de leurs signifiants sont réintroduits dans la langue avec de nouveaux sens. Ce phénomène est beaucoup productif en français avec des exemples qui se rencontrent à travers les âges.

Grevisse et Goose (1980: 61) distinguent quatre méthodes principales à travers lesquelles le changement de sens a lieu à savoir la restriction de sens, l'extension de sens, la métonymie et la métaphore (sens figuré). Ex : pondre aujourd'hui signifie déposer des oufs mais avant signifiait déposer n'importe quoi (restriction sémantique); souris passe d'un animal à un emploi en informatique (extension sémantique), la clé du problème (la clé n'est plus un métal (concret) mais une solution (abstrait)) (usage métaphorique).

\section{L'enrichissement par l'emprunt}

L'emprunt est une voie importante pour l'enrichissement lexical et représente souvent la solution la plus viable pour enrichir l'inventaire des éléments lexicaux d'une langue. Enrichir la langue par le procédé d'emprunt consiste à intégrer dans son lexique des mots étrangers. Tout au cours de son histoire, le français a emprunté et continuera à emprunter bien qu'aujourd'hui, il existe des tentatives pour freiner ce processus pour ne pas - dit-on - corrompre la langue. Le français a le plus emprunté aux langues comme le gaulois, l'ancien germanique, l'arabe, l'italien, l'allemand, l'espagnol et aujourd'hui emprunte surtout à l'anglais. Ce tableau nous donne un inventaire de ces mots venus d'autres langues (tirés de Walter, $2005: 36-43$ ) :

\begin{tabular}{|l|l|}
\hline Langues & Emprunts \\
\hline Germanique & bleu, blanc, gris, haie, blé, gazon, renard, chouette, guerre, flèche, haïr, etc. \\
\hline Arabe & algèbre, chiffre, zéro, zénith, alcool, calife, coton, magasin, matelas, etc. \\
\hline Italien & caleçon, pantalon, costume, veste, dessin, alerte, alarme, pommade, colis, etc. \\
\hline Espagnol & camarade, moustique, chocolat, cacao, cacahuète, tornade, cédille, etc. \\
\hline Anglais & star, rail, wagon, manager, stress, boxe, sprint, tennis, etc. \\
\hline Allemand & album, statistique, paranoïa, etc. \\
\hline
\end{tabular}

Ces emprunts parmi tant d'autres sont si bien intégrés dans la langue qu'ils appartiennent maintenant au patrimoine français et ils peuvent contribuer davantage à la productivité ou à l'enrichissement de la langue à travers les procédés étudiés précédemment. Illustrons ce fait avec les exemples suivants tirés du dictionnaire $L e$ 
Petit Larousse Illustré 2012:

\begin{tabular}{|l|l|}
\hline \multicolumn{1}{|c|}{ Emprunts } & \multicolumn{1}{c|}{ Néologismes } \\
\hline al-djabr (arabe) - algèbre (français) & algébrique, algébriquement, algébriste \\
\hline al-kuhl (arabe) - alcool (français) & alcoolémie, alcoolique, alcoolisable, alcoolisme \\
\hline té (chinois) - thé (français) & théier, théière, théine \\
\hline wagon (anglais) - wagon (français) & wagonnette, wagon-restaurant, wagon-citerne, \\
\hline boycott (anglais) - boycott (français) & boycottage, boycotter, boycotteur \\
\hline
\end{tabular}

\section{L'intervention officielle}

Par intervention officielle, on entend la création de nouveaux mots par l'intervention de l'Etat. Il existe en effet des commissions terminologiques dont la tâche est de produire de nouveaux mots pour désigner de nouvelles notions et réalités qui apparaissent. Les néologismes officiels ainsi formés concernent surtout les domaines techniques et scientifiques. Le français ne fait pas exception à cette pratique. Il existe en son sein des commissions spécialisées de terminologie et de néologie.

La création de ces commissions de terminologie et de néologie est surtout pour combattre l'invasion de mots étrangers dans la langue et pour montrer que le français en tant que langue vivante dispose de moyens pour désigner les nouvelles réalités contemporaines dans les domaines technique, scientifique et économique. Fagyal et al. (2006: 146) remarquent de ce fait que "Official neologisms have been created by terminological commissions, in the hope of fending off foreign terms that have entered the French language in France and other French-speaking countries". Dans cette conception, le néologisme officiel se présente comme un terme recommandé par le législateur à la place d'un terme étranger. En exemples de ce genre de mots créés par la commission de terminologie française, on peut citer : baladeur qui remplace walkman; logiciel qui remplace software; parrainage qui remplace sponsorship; courriel qui remplace e-mail.

Après ce tour d'horizon sur des différents moyens à la disposition du français pour l'enrichissement de son lexique, tournons-nous maintenant vers le yoruba.

\section{Le lexique du yoruba : Modes d'enrichissement}

Le yoruba est une langue agglutinative-isolante. Ce statut a un impact sur son profil lexical. Awoyale (en ligne) remarque que le yoruba est isolant dans son système verbal et agglutinant dans son système nominal. Une conséquence de cette assertion est que la majorité des verbes en yoruba sont monosyllabiques et monomorphémiques et que la majorité des noms, par contre, sont polysyllabiques et polymorphémiques et ces noms sont en majorité construits (Fadipe, 1970 : 57).

Afin de traduire effectivement l'évolution du monde, maintenir son statut de langue vivante et être de pair avec les autres langues du monde, le yoruba doit enrichir son lexique. Comme toute langue, il est doté de mécanismes d'enrichissement lexical. Nous traiterons les modes d'enrichissement du lexique du yoruba sous les mêmes rubriques comme nous l'avons fait pour le français.

\section{A. L'enrichissement lexical par des procédés morphologiques}

Les principaux procédés morphologiques d'enrichissement lexical identifiés en yoruba sont: la dérivation, la composition et la réduplication.

\section{(a) La dérivation}

Quand on parle de dérivation, on se réfère aux procédés comme la préfixation, l'infixation et la suffixation qui impliquent l'ajout d'un affixe soit au début, au sein ou à la fin d'un mot respectivement pour avoir un nouveau mot.

Dans une recherche, Oshounniran (2008: 47) classait le yoruba parmi les langues dérivationnelles à relations simples. Avec ces langues, a-t-il expliqué, il est possible de modifier la signification des éléments radicaux à travers la dérivation ou la modification interne des radicaux. Ainsi, en yoruba, seule la préfixation et l'infixation sont utiles dans l'enrichissement lexical. La suffixation comme procédé morphologique dérivationnel n'y a pas droit de cité.

La préfixation est l'un des procédés les plus productifs dans la création lexicale en yoruba. Les préfixes ici sont des voyelles. Toutes les voyelles orales du yoruba (il y en a sept) sauf $/ \mathrm{u} /$ peuvent être utilisées comme préfixes pour dériver de nouveaux mots. Le yoruba est une langue à ton et ces tons sont portés - à part les consonnes nasales- par des voyelles. En conséquence, les six voyelles-préfixes (a, e, ẹ, o, ọ, i) associées aux tons (ton moyen (') et ton tombant (')) nous donnent deux formes pour chacune des voyelles, enrichissant ainsi davantage la gamme de créations possibles. Il faut noter que le ton montant (') ne se met pas sur une voyelle initiale du mot en yoruba (sauf peut-être dans les emprunts), il ne vient donc pas enrichir les possibilités vocaliques préfixables. Ainsi, avec les tons, nous avons douze voyelles-préfixes qui sont (avec des exemples illustratifs):

$$
\text { - a : a + kọ̀wé (écrire) - akọ̀wé (écrivain) }
$$


- $\quad$ à : à + jọ (ressembler)

- $\quad \mathrm{i}: \mathrm{i}+$ yọ ( se réjouir)

- $\quad$ ì : ì + gbàgbọ́ (croire)

- o : o + dì (attacher)

- $\quad$ ò : ò + kú (mourir)

- e : ẹ + rù (porter)

- $\quad$ è : è̀ + gún (percer)

- e : e + wè (emballer)

- $\quad$ è : è + tò (arranger)

- o : o + dẹ (chasser)

- $\quad$ ò : ò̀ + dájú (être sans pitié)
- àjọ (association)

- iyọ (sel)

- ìgbàgbọ́ (la foi)

- odì (malveillance)

- òkú (cadavre)

- ẹrù (bagage)

-ègún (épine)

- ewè (feuille)

- ètò (ordre)

- odẹ (chasseur)

- òdájú (personne sans pitié)

Ces voyelles-préfixes sont tous des déverbo-nominaux (Voyelle-préfixe $+\mathbf{V}=\mathbf{N}$ ). Ils aident tous à créer des noms. A part ces voyelles-préfixes, il existe d'autres préfixes qui servent à la formation des noms tels que 'àti', 'àì' et 'oní', 'òn'

- 'àti', 'àì', 'òn' sont principalement des déverbo-nominaux, ils s'ajoutent à un verbe pour donner un nom (àti/àì/òn $+\mathbf{V}=\mathbf{N})$.

$$
\begin{array}{lll}
\text { Ex : } & \text { àti + lọ (aller) } & \text { - àtilọ (le départ) } \\
& \text { àì + lówó (avoir de l'argent) } & \text { - àilówó (la pauvreté) } \\
& \text { òn + dè (attacher) } & \text { - òndè (un prisonnier) }
\end{array}
$$

- 'onì' est un dénomino-nominal, il s'ajoute à un nom pour donner un autre nom $($ oní $+\mathbf{N}=\mathbf{N})$. Ex : oní + kẹ̀kẹ̀ (vélo) - oníkẹ̀kẹ̀ (possesseur d'un vélo) oní + ìdajọ (jugement) - onídàjọ (le juge)

Avec le préfixe 'oní' dans la formation du nom, 'n' devient 'l' devant les voyelles /a, e, ẹ, o, ọ/ et avec ces voyelles, le 'o' de 'oní' est remplacé par la voyelle initiale du mot de base (Jeje, 1979 : 82). Ainsi devant ces voyelles, 'oní' devient : consonne initiale du mot de base + I + mot de base. Exemples :

$$
\begin{array}{ll}
\text { oní }+ \text { ẹkọ (bouillie) } & \text { - ẹlẹkọ (vendeur de bouillie) } \\
\text { oní }+ \text { epo (huile) } & \text { - elépo (vendeur d'huile) }
\end{array}
$$

On parlera de conversion ou de dérivation impropre lorsque la création lexicale implique seulement un changement de classe grammaticale. Le yoruba en fait aussi usage surtout entre l'adjectif et le nom. Ex : Adjectif devient Nom : Kékeré ni mo fẹ (C'est le petit que je veux); Nlà yen dara (Ce gros est bon); Dudu yi wu mi (Ce noir me plaît).

\section{(b) La composition}

Le yoruba se sert aussi de la composition, c'est-à-dire de la juxtaposition de deux bases lexicales pour former un nouveau mot: $\left(M_{1}\right)+\left(M_{2}\right)=\left(M_{3}\right)$.

Ex : $\quad$ owó + orí = ówóorí (l'argent + la tête $=$ l'impôt $)$ ojọ + ọla $=$ ojọọla $($ le jour + demain $=$ le futur $)$

Parmi les types de combinaison possibles, on distingue :

Nom + Nom $=$ Nom :

Verbe + Nom $=$ Verbe :

Verbe + verbe $=$ Verbe :

Préposition + Nom $=$ Préposition :

Nom + Adjectif $=$ Nom :

$$
\begin{aligned}
& \text { ẹran }+ \text { oko }=\text { ẹranko }(\text { la viande }+ \text { la brousse }=\text { animal }) \\
& \text { pa }+ \text { inà }=\text { panà }(\text { éteindre }+ \text { le feu }=\text { éteindre le feu }) \\
& \text { pa }+ \text { dà }=\text { padà }(\text { mourir }+ \text { être }=\text { retourner }) \\
& \text { ní }+ \text { inú }=\text { nínú }(a ̀ \text { le ventre }=\text { dans }) \\
& \text { iyè }(\text { mémoire })+\text { mejì }(\text { deux })=\text { iyèméjì }(\text { le doute })
\end{aligned}
$$

La nature agglutinative de la langue peut lui permettre de juxtaposer les éléments de toute une phrase ou de toute une proposition pour produire un nouveau mot :

Ex : $\quad$ Adé + wá + sí + ilé $=$ Adéwálé $(\mathrm{N}+\mathrm{V}+$ Prép $+\mathrm{N}=$ Nom propre $)$

(La couronne est venue à la maison)

Mo + tùn + rí + ayọ $=$ Motùnrayọ $($ Pron. + Adv. $+\mathrm{V}+\mathrm{N}=$ Nom propre $)$

(J'ai vu encore le bonheur)

Ce procédé est très utile surtout dans la création de noms propres.

La composition en yoruba est associée à certaines réalités morphophonologiques dont:

- La possibilité d'assimilation vocalique à la jointure. Ici, on parlera plutôt d'harmonisation vocalique. On peut donner ces exemples : ilé + iṣẹ́ $=$ iléesẹ $($ maison + travail $=$ lieu de travail) $;$ ará + ilú $=$ aráàlú (peuple 
+ village $=$ villageois $)$.

- L'élision c'est-à-dire la disparition d'une ou de plusieurs voyelles à la jointure : ẹran + oko $=$ ẹranko $($ viande + brousse $=$ animal $) ;$ jẹ + ogùn $=$ jogún $($ manger + héritage $=$ hériter $)$.

\section{(c) La réduplication}

Comme nous l'avons dit à propos du français, la réduplication est un procédé qui consiste à redoubler un mot ou une partie d'un mot pour former un autre mot. On peut considérer cette pratique comme une autre forme de composition mais où les deux mots en juxtaposition sont identiques $\left(\mathrm{M}_{1}+\mathrm{M}_{2}=\mathrm{M}_{3}\right.$ avec $\left.\mathrm{M}_{1}=\mathrm{M}_{2}\right)$. Ce procédé est beaucoup plus productif en yoruba. La réduplication est qualifiée de partielle si c'est seulement une partie du même mot (en yoruba, la première ou la dernière syllabe du mot) qui est répétée et totale, quand c'est le mot entier qui se répète. La réduplication en tant que procédé morphologique peut conduire à la création de nouveaux mots et cela est possible avec toutes les classes grammaticales.

\section{- La réduplication partielle}

Dans la réduplication partielle, la partie répétée (première ou dernière syllabe du mot) peut être antéposée ou postposée au même mot.

Antéposée : La première syllabe est répétée et placée avant le même mot. gbọ + gbọọọ $($ adj) $=$ gbọgbọọọ $($ adj) (très long)

Cela ne change pas la classe grammaticale du mot. Nous n'avons pas trouvé de cas où la dernière syllabe est répétée et antéposée au mot de base.

Postposée : La première syllabe est répétée et postposée au même mot.

tààrà $(\mathrm{adv})+$ tà $=$ tààràtà $(\mathrm{adv})$ (tout droit)

wẹerẹ $(\mathrm{adv})+$ wẹ $=$ wẹerẹwẹ (adv) (très aisément)

La dernière syllabe est répétée et postposée au même mot

tààrà $(\mathrm{adv})+$ rà $=$ tààràrà $(\mathrm{adv})$ (tout droit)

geere $(\mathrm{adv})+\mathrm{re}=$ geerere $(\mathrm{adv})($ très droit $)$

La réduplication partielle du verbe, en particulier, conduit dans la quasi-totalité des cas à une nouvelle classe grammaticale, surtout aux noms : lọ (aller) - lilọ (le départ) ; jẹun (manger) - jijẹun (le repas). Ici, c'est la consonne initiale du mot qui est répétée, le /i/ est permanent et joue le rôle d'infixe de jointure qui aide à la formation d'un nom. Awobuluyi (cité par Táíwo 2009: 47) considère ces consonnes répétées comme des consonnes-préfixes (consonantal prefix) et admet que toutes les consonnes du yoruba peuvent être ainsi copiées. Ces consonnes préfixes changent la classe grammaticale du nouveau mot construit. En voici d'autres exemples : gbọ (V) (entendre) - gbigbọ (N) (la compréhension) ; bọ (V) (arriver) - bibọ (N) (le retour) ; ga (V) (grandir) - giga (N) (la hauteur).

La réduplication partielle avec le nom aide à exprimer la répétition comme dans les exemples suivants : ojúmọ $(\mathrm{N})($ le jour $)$ - ojoojúmọ $(\mathrm{N})($ chaque jour) ; àáọ̀ $(\mathrm{N})($ le matin) - àràáọ̀ $(\mathrm{N})$ (chaque jour) ; ọdún $(\mathrm{N})$ (l'année) - ọdọọdún (N) (chaque année).

\section{- La réduplication totale}

Ici, toutes les classes grammaticales peuvent être impliquées. La réduplication totale nominale connote le degré ou l'intensité, la réduplication totale verbale, le nom d'une agence et la réduplication totale adjectivale, l'intensité ou la pluralité. Ex :
Nominale :
ọmọ + ọmọ
= ọmọomọ $($ enfant + enfant $=$ petit-fils $)$
Verbale: $\quad$ paná + paná
= panápaná (éteindre le feu $\mathrm{x} 2=$ pompier)
Adjectivale :
nlà + nlà
$=$ nlànlà $($ gros + gros $=$ de gros $)$

La réduplication totale peut aussi entrainer un changement de classe grammaticale, surtout au niveau des verbes.

$$
\begin{array}{lll}
\text { Ex : } & \text { pẹja }+ \text { pẹa }(V) & =\text { pẹjapẹja }(N)(\text { pêcher }+ \text { pêcher }=\text { pêcheur }) \\
& \text { jagun }+ \text { jagun }(V) & =\text { jagunjagun }(\mathrm{N})(\text { combattre } \times 2=\text { soldat })
\end{array}
$$

\section{- La réduplication par infixation}

Le yoruba possède certains infixes qui peuvent entraîner une nouvelle création lexicale. Mais l'infixe ne se fixe pas au sein d'un mot mais plutôt à la jointure d'un même mot qui est redoublé. On pourrait en toute logique qualifier ce processus de réduplication par infixation : le même mot est redoublé mais le tout est joint à l'aide d'un infixe. Parmi d'autres infixes qui peuvent jouer ce rôle en yoruba sont : ki, kú, di/de, dé, ni, sí. 


$$
\begin{aligned}
& \text { ọmọ }+ \text { ki }+ \text { ọmọ = ọmọọmọ (tout enfant/mauvais enfant) } \\
& \text { ìnìn }+ \text { kù }+ \text { ìrìn } \quad=\text { ìrìnkurìn (vagabondage) } \\
& \text { iran }+ \text { de }+ \text { iran } \quad=\text { iranderan (de génération en génération) } \\
& \text { ọwọ }+ \text { dé }+ \text { ọwọ } \quad \text { = ọwọdọwọ (de main en main) } \\
& \text { àgbà }+n i+\text { àgbà = àgbàlagbà (vieux) } \\
& \text { (N'oubliez pas que ' } n \text { ' devient 'l' devant la voyelle orale /a/) } \\
& \text { ojú }+ \text { sí }+ \text { ojú }=\text { ojúsójù (face à face) }
\end{aligned}
$$

Les phénomènes d'harmonisation vocalique, d'élision et de déplacement ou de changement de ton rentrent en ligne de compte où cela est nécessaire.

\section{B. L'enrichissement lexical par le sens}

Le yoruba aussi travaille sur le sens dans la tentative de trouver de nouvelles dénominations à de nouveaux concepts. La figure de style prédominante dans ce processus en yoruba est la métaphore. Le yoruba procède à une extension sémantique de mots existants pour couvrir d'autres concepts qui leur sont métaphoriquement liés. Fadipe (1970:58) remarque que "The figurative extension of the use of word to cover other meanings besides that for which it was originally coined is carried much further in Yoruba than in European Languages". Il donne l'exemple des parties du corps : 'orí', 'ojú', 'ẹnu', etc. dont les sens ont été métaphoriquement utilisés pour désigner d'autres concepts : orìigi - le dessus du bois ; ojúòkun - la surface de la mer ; ojù-ibọ - lieu de sacrifice, etc. Et de cette manière, toutes les parties du corps, en exemple ici, peuvent avoir leur sens élargi figurativement à d'autres concepts.

Le yoruba procède par association métaphorique + paraphrase lexicale pour dénommer d'autres concepts. On peut prendre l'exemple de 'la télévision' que le yoruba dénomme 'erọ amohun maworan' (machine qui transmet le son et montre l'image), de l'avion 'ọkọ-òfurufú' (moyen de transport qui vole dans l'air), de la radio 'asọrọ magbesi' (celui qui parle et qui n'attend pas de réponse), de l'hélicoptère 'ọkọ aba sibi owu' (moyen de transport qui descend où il veut). A travers ce procédé, le yoruba a la capacité de trouver une dénomination à tout concept.

\section{L'enrichissement lexical par l'emprunt}

Le yoruba abonde aussi en emprunts. Il emprunte principalement à l'hausa, à l'anglais et à l'arabe (souvent via l'hausa), toutes étant des langues de son environnement immédiat. Les interactions politiques, commerciales, religieuses, entre autres, ont favorisé l'entrée des emprunts dans le lexique yoruba. Quant à la fortune de ces mots étrangers dans la langue, Adeyemo (1998:35) note que "Some words have come and live but disappear shortly at will after sometime whereas some words have come and stayed permanently". Ainsi, nous avons des emprunts qui se sont totalement intégrés jusqu'à faire partie de son patrimoine lexical. Le tableau suivant nous donne un panorama de la répartition de ces emprunts, selon leurs origines, que nous tirons d'Ogunbowale (1970: 189-191).

\begin{tabular}{|l|l|}
\hline Langues & \multicolumn{1}{c|}{ Emprunts } \\
\hline Anglais & gómìnà, kúáyà, búrẹdì, kàlẹndà, kápẹẹtì, kúlà, rìsiitì, sínimá, páànu, rélùwéè \\
\hline Hausa & filà, àlùbọsà, àlààfià, wàhàlà, lábárè, gbúgbúrú, námà, ràgó, báárà \\
\hline Arabe & Kálàmú, dúníyàn, sànmà, mọsálási, jímọ \\
\hline Français & pọtimátò (via l'anglais) \\
\hline
\end{tabular}

Les emprunts sont aussi dynamiques qu'en yoruba, ainsi intégrés, ils participent à la création de nouvelles unités lexicales à travers des procédés morphologiques d'enrichissement lexical : bread (anglais) búrẹdì - onibúrẹdì ; albasa (hausa) - alubọsa - alalubọsa ; jumua (arabe) - jímọ - jímọjímọ.

Dans le langage des jeunes, on rencontre la création de mots nouveaux à partir des emprunts par le procédé de la troncation et ceci après adaptation phonologique de l'emprunt au système du yoruba. Des cas rencontrés sont des cas de troncation par apocope : vulganizer - fọganaiza - fọga ; mechanic - mẹkaniiki mẹko ; certificate - satifiketi - sati ; original - ọrijina - ọrijo.

\section{L'intervention officielle}

Le yoruba n'est pas une langue imposée par l'État, c'est-à-dire à statut officiel, elle n'est qu'une langue régionale. Il n'existe pas d'organisme officiel régulateur de la langue. En considérant l'intervention officielle comme étant la création de nouveaux mots par l'intervention de l'État ou sanctionnés par l'État, on déduira qu'en yoruba, il n'y a pas de néologismes officiels. 


\section{Conclusion}

Ce voyage au cœur linguistique du français et du yoruba nous a donné un aperçu des outils à la disposition des deux langues pour répondre efficacement à l'évolution de leur société respective. Le tableau suivant nous résume les mécanismes d'enrichissement lexical attestés en français et en yoruba.

\begin{tabular}{|c|c|c|c|c|}
\hline \multicolumn{3}{|c|}{ Mécanismes } & \multirow{2}{*}{$\begin{array}{c}\text { Français } \\
+\end{array}$} & \multirow{2}{*}{$\frac{\text { Yoruba }}{+}$} \\
\hline \multirow{12}{*}{$\begin{array}{l}\text { Enrichissement par les } \\
\text { procédés morphologiques }\end{array}$} & \multirow[t]{5}{*}{ La dérivation } & préfixale & & \\
\hline & & suffixale & + & - \\
\hline & & parasynthétique & + & - \\
\hline & & régressive & + & + \\
\hline & & impropre & + & + \\
\hline & \multicolumn{2}{|l|}{ La composition } & + & + \\
\hline & \multicolumn{2}{|l|}{ La troncation } & + & + \\
\hline & \multicolumn{2}{|l|}{ La siglaison } & + & - \\
\hline & \multicolumn{2}{|l|}{ L'acronymisation } & + & - \\
\hline & \multirow[t]{3}{*}{ La réduplication } & partielle & + & + \\
\hline & & totale & + & + \\
\hline & & par infixation & - & + \\
\hline \multicolumn{3}{|l|}{ Enrichissement par le sens } & + & + \\
\hline \multicolumn{3}{|c|}{ Enrichissement par l'emprunt } & + & + \\
\hline \multicolumn{3}{|l|}{ L'intervention officielle } & + & - \\
\hline
\end{tabular}

Ce tableau nous permet de tirer les conclusions suivantes :

$>$ Parmi les procédés disponibles en yoruba, seule la réduplication par infixation n'est pas attestée en français,

$>$ Les procédés comme la dérivation suffixale, la dérivation parasynthétique, la siglaison, l'acronymisation et l'intervention officielle, présents en français, ne sont pas attestés en yoruba,

$>$ Alors que la dérivation est le procédé morphologique le plus productif en français, c'est la réduplication qui semble jouer ce rôle en yoruba,

$>$ Au niveau des procédés morphologiques, les règles morphologiques qui déterminent la manière dont les morphèmes se lient pour donner de nouveaux mots diffèrent de manière significative dans les deux langues d'autant plus que le français est une langue flexionnelle alors que le yoruba est largement isolante.

\section{Bibliographie}

[1]. Adeyemo, B. (1998) : Yorùbá as a Language of an Immediate Environment, Abeokuta : GOAD Educational Publisher.

[2]. Awóbùlúyì, O. (2001): "Mọfọoọi Ėdè Yorùbá" In Àjàyí, Bádé (ed.) : Ėkọ ijìnlẹ Yorùbá : Edá - Ėdè, Lítíréṣo àti Àsạà, Ilorin : Unilorin, Department of Linguistics and Nigerian Languages, pp.47-70.

[3]. Awoyale, Y. "Global Yoruba Lexical Database v. 1.0", http://www.ldc.upenn.edu/Catalog/docs/LDC2008L03/Global_Yoruba_ Lexical_Database.pdf Consulté le 22/7/2012

[4]. Bamgbose, A. (1967): A Short Yoruba Grammar, Ibadan: Heinemann Educational Books (NIG) Ltd.

[5]. Fadipe, N. A. (1970): The Sociology of the Yoruba, Ibadan: University Press.

[6]. Fagyal, Z. et al. (2006): French: A Linguistic Introduction, United Kingdom: Cambridge University Press.

[7]. Genouvrier, E. \& Peytard, J. (1970) : Linguistique et enseignement du français, Paris : Librairie Larousse.

[8]. Grevisse, M. et Goose, A. (1980) : Nouvelle grammaire française, Paris : Duculot.

[9]. Huot, H. (2001) : Morphologie : Forme et sens des mots du français, Paris : Armand Colin.

[10]. Jeje, A. (1979) : Yorùbá fún Ilé Èkó Gíga, Ibadan, Heinemann Educational Books (NIG) Ltd.

[11]. Le Petit Larousse Illustré (2012), Paris : Larousse.

[12]. Le Robert \& Nathan (1995) : Grammaire, Paris, Nathan.

[13]. Lessard, G. (1996) : FREN 215 : Introduction à la linguistique française, http://post.queensu.ca/ lessardg/Cours/215/chap1.html

[14]. Martinet, A. (1970): Eléments de linguistique générale, Paris : Armand Colin

[15]. Mounin, G. (1974): Dictionnaire de la linguistique, Paris : Presses Universitaires de France.

[16]. Ogunbowale, P. O. (1970): The Essentials of the Yoruba Language, London: University of London Press Ltd.

[17]. Oshounniran, T. A. (2008) : "Les ressources morphosyntaxiques de la pluralisation en français et en yoruba". Mémoire de maitrise non publié, Département de français, Université d’Ilorin.

[18]. Oshounniran, T. A. (2013) : "Les emprunts de source anglaise en français et en yoruba : Morphologie et fonctionnement". Thèse de doctorat présenté au Département de français, Université d’Ilorin.

[19]. Phelizon, J. F. (1976): Vocabulaire de la linguistique, Paris, Editions Rondil.

[20]. Picoche, J., Marchello-Nizia, C. (1991): Histoire de la langue française, Paris, Nathan.

[21]. Táíwò, O. (2009) : "Headedness and the Structure of Yorùbá Compound Words", Taiwan Journal of Linguistics, Vol. 7.1, pp. 2752.

[22]. Walter, H. (2005) : «L'intégration des mots venus d'ailleurs », Alsic : Apprentissage des Langues et Systèmes d'Information et de Communication, vol.8, no.1/2005, pp. 35-44. 